\section{Summary}

A basic difference in the endoplasmic reticulum organization of two absorbing cells responsive to vasopressin

The endoplasmic reticulum has been generally - if not totally ignored in representations of cell transport phenomena. Yet the endoplasmic reticulum (ER) shows a well organized network in most transporting epithelial cells, one odd exception being the principal cells of the mammalian kidney collecting tubule. Bladders of Bufo marinus, widely used as a model of the latter cells, were fixed by immersion in glutaraldehyde $2.5 \%$, followed by $\mathrm{OsO}_{4}$ impregnation for three days. Thick sections $(0.3 \mu \mathrm{m})$ were examined with a transmission electron microscope at $80-100 \mathrm{kV}$. Surprisingly granular cells show a rich transcytoplasmic network of canaliculi having a diameter of 10 to $30 \mathrm{~nm}$, extending from the apical to the basilar membrane and connected to the nuclear sac (figure 1). This impressive endoplasmic reticulum organization - a new finding - is in contrast with the rudimentary aspect of the endoplasmic reticulum of principal cells of mammalian kidney (figure 2) which is made of few vesicular elements also osmiophilic.

Remerciements

Les auteurs désirent remercier le Dr Georges Thiéry pour ses précieux conseils, $M$. Jacques Bernier et Mme Christiane Laurier pour leur collaboration technique. Ce travail a été effectué grâce à une subvention du Conseil de recherches médicales du Canada (MT-2862).

\section{ADRESSE}

K. Danechi : docteur ès sciences, $\mathrm{M}$. Bergeron : professeur et directeur. Département de physiologie, faculté de médecine, université de Montréal, C.P. 6128, Succursale A, Montréal H3C 3J7, Québec, Canada.

$\mathrm{m} / \mathrm{s} n^{\circ} 10$ vol. 5 , décembre 89

\title{
Différence entre les réticulums endoplasmiques de deux types de cellules sensibles à la vasopressine
}

\author{
Kaveh Danechi, Michel Bergeron
}

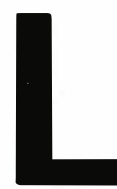

e réticulum endoplasmique est en général, pour ne pas dire toujours, absent des schémas illustrant le transport transépithélial. Pourtant il présente, au niveau des cellules du néphron proximal et du jéjunum, un riche réseau continu possédant les mêmes caractéristiques : des lames fenêtrées enserrant les mitochondries et des canalicules transcytoplasmiques reliés au noyau et contigus aux membranes apicale et basolatérale. L'ampleur de ce réseau, déjà soupçonnée dans les premières études en microscopie électronique [1], a été récemment mieux révélée grâce aux techniques d'imprégnation métallique combinées à la stéréomicroscopie [2-7] ou encore à d'autres techniques histochimiques [8]. La richesse de son organisation et les similitudes morphologiques du réticulum endoplasmique dans des cellules différentes mais douées des mêmes fonctions d'absorption suggèrent qu'il joue un rôle insoupçonné dans le transport des ions ou des métabolites à travers ces épithéliums. L'absence d'un tel réseau dans les cellules principales du tube collecteur du rein [7, 9] devient intrigante, car elles jouent un rôle capital dans le transport de l'eau et des électrolytes sous l'effet de la vasopressine. Nos connaissances sur la liaison membranaire de la vasopressine et sur les mécanismes cellulaires aboutissant à la perméabilité de la membrane apicale proviennent d'un modèle abondamment utilisé, la vessie de crapaud [10, 11]. Il devient dès lors intéressant de vérifier l'organisation et le rôle du réticulum endoplasmique dans cet épithélium. Les résultats que nous présentons montrent que la vessie de crapaud, contrairement à la cellule principale du tube collecteur dont elle est un modèle très utilisé, possède un réticulum endoplasmique bien organisé que révèle l'imprégnation osmique. Des fragments de vessie sont prélevés chez des crapauds gardés dans un bassin d'eau douce depuis 48 heures et ce, dans le but de minimiser la production endogène de vasopressine. Les fragments de vessie sont placés dans une solution de Ringer pour amphibiens [12], puis sont fixés par immersion [6] dans une solution tamponnée de glutaraldéhyde $(2,5 \%)$ et de citrate de calcium pendant 15 minutes. Le tissu est alors lavé et postfixé dans une solution de tétroxyde d'osmium 1,3\% (P/V) tamponnée avec du barbital de sodium à $0,2 \mathrm{M}$. L'imprégnation au tétroxyde d'osmium est ensuite faite durant 4 jours selon les techniques antérieurement décrites $[2,5,6,7]$. Après enrobage, des coupes épaisses de $0,3 \mu \mathrm{m}$ sont observées en microscopie électronique $(80-100 \mathrm{kV})$.

Le réticulum endoplasmique des cel- 
lules granulaires de la vessie de Bufo marinus se compose d'un vaste réseau de canalicules dont le diamètre varie de 10 à $30 \mathrm{~nm}$ mais aussi de 3 à 8 citernes parallèles situées à proximité du noyau, le plus souvent latéralement mais parfois dans la zone supranucléaire (figure 1). Ces citernes en série sont nettement différentes de celles de l'appareil de Golgi. Le réseau de canalicules s'étend dans tout le cytoplasme et présente plusieurs points d'attache avec la membrane nucléaire dont le feuillet externe est aussi fortement imprégné d'osmium, tout comme l'appareil de Golgi. Quoique la continuité entre les canalicules du réticulum endoplasmique et la membrane plasmique ne puisse être démontrée avec certitude, on observe fréquemment des contacts entre les canalicules et les membranes apicales ou basolatérales de la cellule (figure 1). Les canalicules du réticulum endoplasmique enserrent la plupart des mitochondries comme dans les cellules intercalaires du rein de rat $[5,7]$. Les granules, caractéristiques des cellules dites "granu- laires », sont présents du côté apical mais ne sont jamais imprégnés par l'osmium.

Par la présence de granules et d'un riche réticulum endoplasmique, la cellule granulaire de la vessie de crapaud est morphologiquement différente de la cellule principale du tube collecteur du mammifère (figure 2). En effet, la cellule principale du néphron distal n'est dotée chez le rat que d'un réticulum endoplasmique rudimentaire formé de quelques éléments vésiculaires isolés imprégnés d'osmium [7, 9].

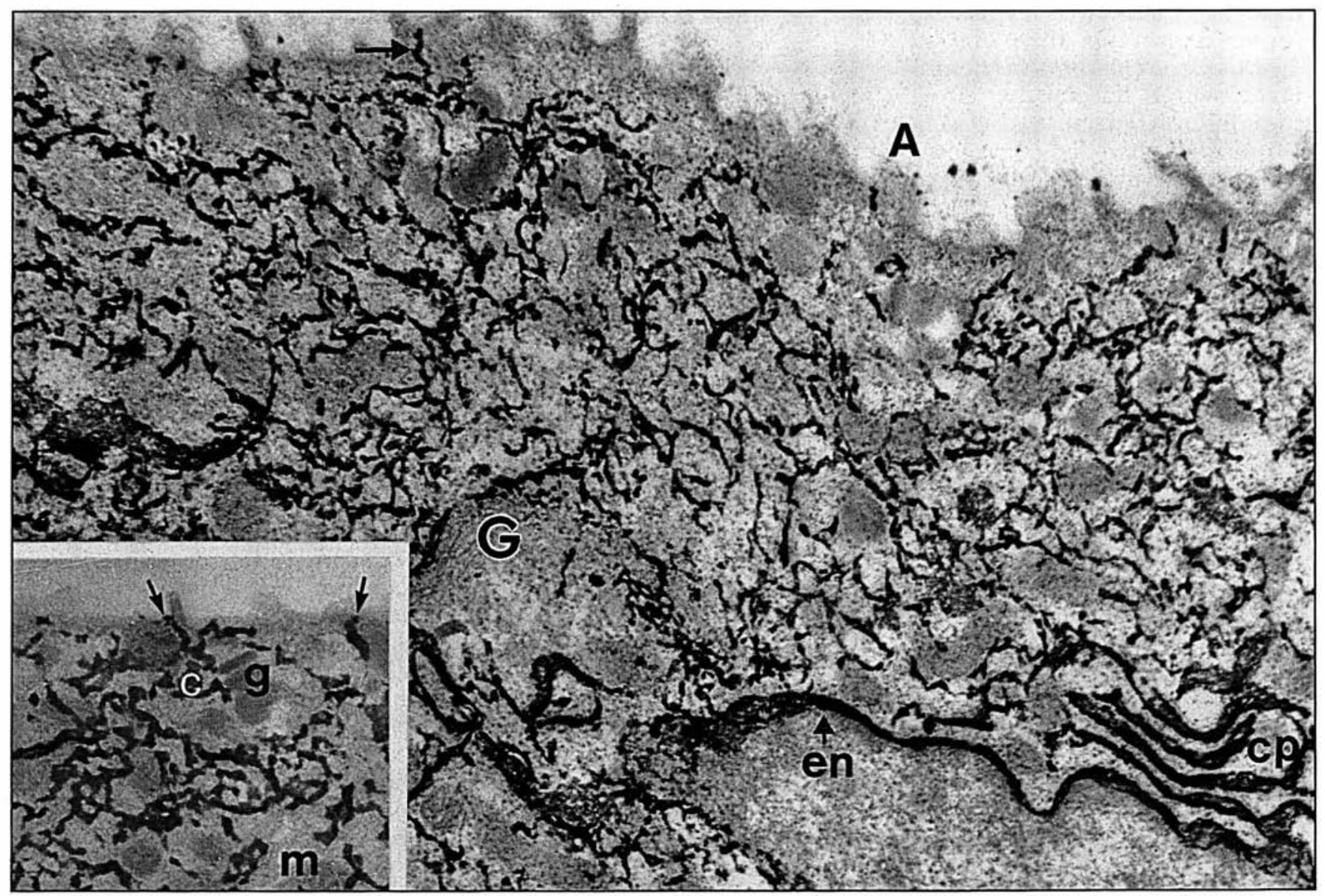

Figure 1. Coupe transversale d'une cellule granulaire de la vessie de Bufo marinus. La vessie a été fixée après une incubation de 30 minutes dans une solution de Ringer standard. Les canalicules du réticulum endoplasmique (c) qui parcourent l'ensemble de la cellule sont en continuité avec les citernes parallèles (cp) et la membrane nucléaire externe (en) qui semble donner origine au réticulum endoplasmique au cours de l'ontogénie [13]. Comme le montre l'encart, à différents endroits, ces canalicules sont apparemment en contact (flèches) avec la membrane plasmique du côté apical (A) (côté urinaire). Plusieurs mitochondries (m) sont enserrées par les canalicules du réticulum endoplasmique. L'appareil de Golgi (G) sur cette photographie est en partie imprégné. A noter les granules typiques de cette cellule (g). Section de 0,3 $\mu m$; $\times 32000$. Encart, $\times 60000$. 


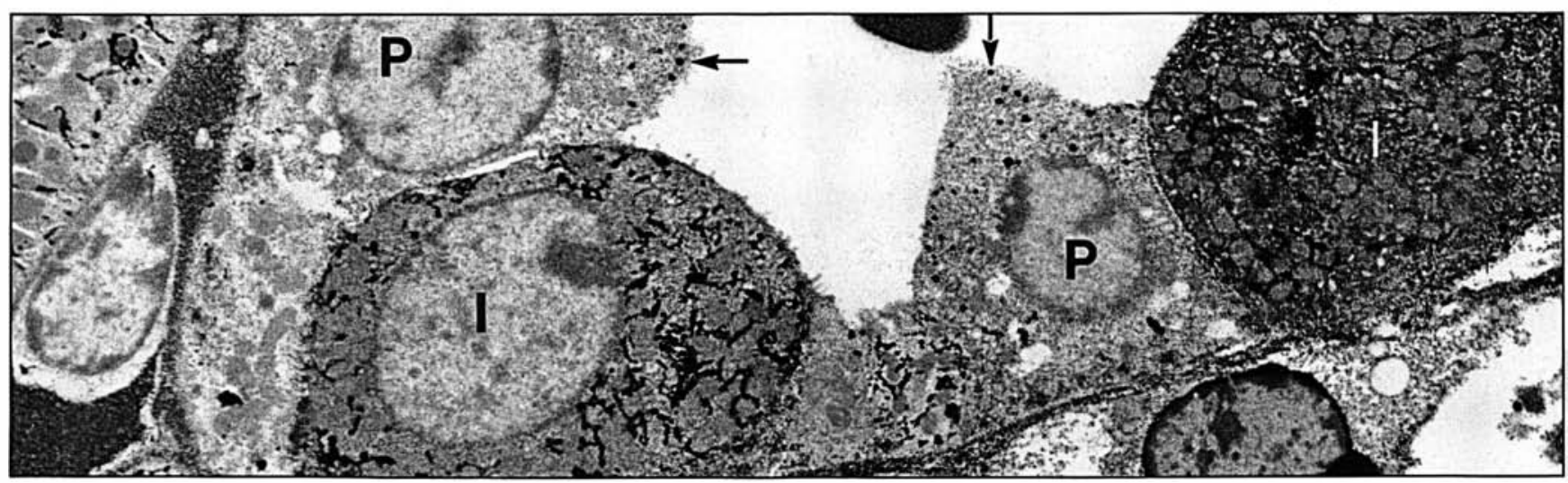

Figure 2. Coupe épaisse $(0,3 \mu \mathrm{m})$ transversale du tube collecteur du rein de rat. L'imprégnation osmique révèle un réticulum endoplasmique développé dans les cellules intercalaires (I) qui correspondent aux cellules riches en mitochondries de la vessie. Toutefois les cellules principales $(P)$, contrairement à la cellule granulaire du batracien, n'ont pas de réseau organisé de réticulum endoplasmique. Dans ces cellules, il est uniquement composé de quelques éléments vésiculaires épars (flèches) dont les dépôts d'osmium sont le reflet (X 3200$)$.

Les cellules riches en mitochondries de la vessie de crapaud analogues aux cellules intercalaires du tube collecteur [7] possèdent aussi un réticulum endoplasmique bien imprégné ayant des rapports étroits avec les nombreuses mitochondries. Dans les cellules basales, en revanche, seul le saccule périnucléaire s'imprègne d'osmium ; sur ce plan, elles ressemblent aux cellules immatures du rein fotal[13] et des cryptes de l'intestin [6].

Nous avons déjà démontré que le réticulum endoplasmique des cellules du néphron proximal et des cellules sécrétrices de la prostate peut répondre à un stimulus métabolique ou hormonal [14, 15]. Des travaux récents indiquent que la stimulation de la vessie par la vasopressine entraîne, de façon réversible, la disparition de l'imprégnation du réticulum endoplasmique des cellules granulaires [16]. Ces résultats suggèrent une relation entre les propriétés du réticulum endoplasmique et le transport de l'eau et des électrolytes. Il sera intéressant de déterminer comment la cellule principale du tube collecteur du rein remplit une fonction de transport semblable à celle de la cellule granulaire de la vessie et analogue à celle d'autres cellules absorbantes (jéjunum, néphron proximal...) sans toutefois posséder un réticulum endoplasmique aussi développé que celles-ci

\section{RÉFÉRENCES}

1. Porter KR, Palade GE. Studies of the endoplasmic reticulum. III. Its form and distribution in striated muscle cell. J Biophysic Biochem Cytol 1957 ; 3 : 269-99.

2. Thiéry G. Colorations signalétiques électives sur coupes épaisses du réticulum endoplasmique de la chromatine et des surfaces cellulaires. Biol Cell 1979; 35 : 159-64.

3. Bergeron $M$, Guérette $D$, Forget J, Thiéry G. Three-dimensional characteristics of the endoplasmic reticulum of the nephron. A transcellular route. Kidney Int 1978; 14 : $102 \mathrm{~A}$.

4. Mollgaard K, Rostgaard J. Morphological aspects of some transporting epithelia suggesting a transcellular pathway via elements of endoplasmic reticulum. J Membrane Biol $1978 ; 40: 71-89$.

5. Bergeron M, Thiéry G. Three-dimensional characteristics of the endoplasmic reticulum of rat renal tubule cells. An electron microscopy study in thick sections. Biol Cell 1981 ; 42: 43-8.

6. Thiéry G, Gaffiero P, Bergeron M. Threedimensional characteristics of the endoplasmic reticulum in the columnar cells of the rat small intestine. An electron microscopy study in thick sections. Am J Anat $1983 ; 1167$ : 47993.

7. Bergeron M, Gaffiero $P$, Thiéry G. Segmental variations in the organization of the endoplasmic reticulum of the rat nephron. A stereomicroscopic study. Cell Tissue Res $1987 ; 247: 215-25$.

8. Novikoff AB, Spater HW, Quintana N. Transepithelial endoplasmic reticulum in rat proximal convoluted tubule. J Histochem Cytochem 1983; 31 : 656-61.

9. Bergeron $M$, Gaffiero $P$, Berthelet $F$, Thiéry $G$. Interrelationship between organelles in kidney cells of adult and developing rat. Pediatr Nephrol 1988; 2 : 100-7.
10. Abramov M, Beauwens R, Cogan E. Cellular events in vasopressin action. Kidney Int 1987 ; 32 : S56-66.

11. Handler JS. Antidiuretic hormone moves membranes. Am J Physiol 1988; 255 : F37582.

12. Bentley PJ. The effects of neurohypophysial extract on water transfer accross the wall of the isolated urinary bladder of the toad Bufo marinus. J Endocrinol 1958; 17 : $201-9$.

13. Gaffiero P, Bergeron M, Thiéry G. Morphological study of cell organelles during development. 1. The nuclear sac and the endoplasmic reticulum of the rat nephron. Biol Cell 1983 ; 49 : 79-82.

14. Beaudry-Lonergan M, Thiéry G, Bergeron $M$. Osmium impregnation of the endoplasmic reticulum correlates with the functional status of the prostatic secretory cell. Biol Cell 1985; 54 : 181-6.

15. Berthelet F, Beaudry-Lonergan M, Bergeron $M$. Proliferation of the endoplasmic reticulum of the proximal nephron cells during chronic metabolic acidosis and after treatment with triamcinolone. In : Kovacevic $Z$, Guder WG, eds. Molecular Nephrology. Biochemical Aspects of Kidney Function. Berlin, New York: Walter de Gruyter \& Co, 1987 213-9.

16. Danechi K, Bergeron M. Vasopressin modifies endoplasmic reticulum reactivity in toad urinary bladder. Kidney Int 1990; 37 (sous presse).

\section{TIRÉS A PART}

K. Danechi. 